\title{
The regulation of MASPIN expression in epithelial ovarian cancer: Association with p53 status, and MASPIN promoter methylation: A Gynecologic Oncology Group study ${ }^{2}$
}

\author{
Angeles Alvarez Secord ${ }^{\text {a,* }}$, Kathleen M. Darcy ${ }^{\text {b }}$, Alan Hutson ${ }^{\text {b }}$, Zhiqing Huang a , Paula S. Lee ${ }^{\text {a }}$, \\ Elizabeth L. Jewell a , Laura J. Havrilesky ${ }^{a}$, Maurie Markman ${ }^{\text {c}}$, Franco Muggia ${ }^{d}$, Susan K. Murphy ${ }^{\text {a }}$ \\ a Division of Gynecologic Oncology, Department of Obstetrics and Gynecology, Duke University Medical Center, Durham, NC 27710, USA

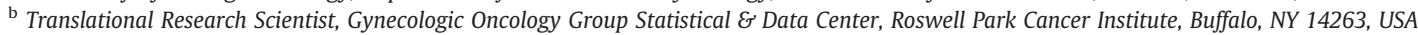 \\ ' Cancer Treatment Centers of America, Eastern Regional Medical Center, Philadelphia, PA 19124, USA \\ ${ }^{d}$ NYU Clinical Cancer Center, New York, NY 10016, USA
}

\section{A R T I C L E I N F O}

Article history:

Received 3 June 2011

Accepted 4 August 2011

Available online 8 September 2011

\section{Keywords:}

MASPIN

Ovarian carcinoma

Methylation

p53

\section{A B S T R A C T}

Objectives. To elucidate the regulation of MASPIN expression in epithelial ovarian cancer (EOC) and associations with $p 53$ status and MASPIN promoter methylation.

Methods. Seven EOC cell lines and 110 advanced stage EOC specimens were analyzed for MASPIN promoter methylation. The cell lines were treated with 5-azacytidine (5-azaC) and evaluated for MASPIN promoter methylation, protein, and mRNA expression. Wild-type (wt) p53 was transiently transfected into the mutant p53 ( $\mathrm{m}$ p53) SKOV3 cells which were treated with 5-azaC. Phosphor imager analysis quantified the percent methylation of the MASPIN promoter.

Results. Of the 3 MASPIN-low $\mathrm{m} p 53$ cell lines 2 had greater than 5\% MASPIN methylation whereas only 1 of 4 MASPIN-high wt $p 53$ cell lines had greater than $5 \%$ MASPIN methylation. Despite the presence of aberrant MASPIN promoter methylation in SKOV3 cells, wt p53-transfection alone resulted in a 3.3-fold increase in MASPIN mRNA. The combination of 5-azaC and wt $p 53$-transfection produced a 36\% reduction in MASPIN promoter methylation and 4.5-fold increase in MASPIN transcription. Among the 110 ovarian cancer specimens analyzed for methylation of the MASPIN promoter, $81.8 \%$ were weakly methylated, $14.5 \%$ were heavily methylated and $3.6 \%$ were fully methylated. There was no relationship between promoter methylation and p53 status or MASPIN protein expression. However, MASPIN protein was 6 times more likely to be detected in cancer specimens that harbor a p53 mutation relative to cancer specimens with a wt p53 gene.

Conclusion. The regulation of MASPIN is a complex multifactorial process that may be controlled by both $p 53$ dependent and -independent epigenetic mechanisms.

(c) 2011 Elsevier Inc. All rights reserved.

\footnotetext{
你 This study was supported by the American Association of Obstetricians and Gynecologists Foundation, the Berlex Scholar Award in Basic Science, and National Cancer Institute grants to the Gynecological Oncology Group (GOG) Administrative Office along with the GOG Tissue Bank (CA 27469) and the GOG Statistical and Data Center (CA 37517). The following institutions participated in this study: University of Alabama at Birmingham, Oregon Health Sciences University, Duke University Medical Center, Abington Memorial Hospital, University of Rochester Medical Center, Walter Reed Army Medical Center, Wayne State University, University of Minnesota Medical School, University of Southern California at Los Angeles, University of Mississippi Medical Center, Colorado Gynecologic Oncology Group P.C., University of California at Los Angeles, University of Washington, University of Pennsylvania Cancer Center, University of Miami School of Medicine, Milton S. Hershey Medical Center, Georgetown University Hospital, University of Cincinnati, University of North Carolina School of Medicine, University of Iowa Hospitals and Clinics, University of Texas Southwestern Medical Center at Dallas, Indiana University School of Medicine, Wake Forest University School of Medicine, Albany Medical College, University of California Medical Center at Irvine, Tufts-New England Medical Center, RushPresbyterian-St. Luke's Medical Center, University of Kentucky, Eastern Virginia Medical School, The Cleveland Clinic Foundation, Johns Hopkins Oncology Center, State University of New York at Stony Brook, Eastern Pennsylvania GYN/ONC Center, P.C., Southwestern Oncology Group, Washington University School of Medicine, Memorial Sloan-Kettering Cancer Center, Columbus Cancer Council, University of Massachusetts Medical School, Fox Chase Cancer Center, Medical University of South Carolina, Women's Cancer Center, University of Oklahoma, University of Virginia Health Sciences Center, University of Chicago, University of Arizona Health Science Center, Tacoma General Hospital, Eastern Collaborative Oncology Group, Thomas Jefferson University Hospital, Case Western Reserve University, and Tampa Bay Cancer Consortium.

* Corresponding author at: DUMC 3079, Division of Gynecologic Oncology, Duke University Medical Center, Durham, NC 27710. Fax: +1919684 8719

E-mail address: secor002@mc.duke.edu (A. Alvarez Secord).
} 


\section{Introduction}

MASPIN, a serine protease inhibitor in the serpin super family of serine protease inhibitors, functions as a tumor suppressor by inhibiting tumor cell motility, invasion, metastasis, and angiogenesis [1-4]. MASPIN has been shown to have differential sub cellular expression in ovarian cancer $[5,6]$ and have prognostic significance for survival [5-7]. Furthermore, transfection of wild-type (wt) MASPIN into aggressive ovarian cancer cell lines has been shown to inhibit the invasive activity of these cells 44-68\% [6]. Although the molecular mechanisms regulating MASPIN expression, down-regulation and loss during ovarian cancer progression are as yet undefined, observations from other cancers suggest that these events will likely be under the control of both normal and aberrant transcriptional and epigenetic mechanisms [8-16].

The p53 tumor suppressor pathway has been implicated in the regulation of MASPIN expression in other solid tumors [8-12] and p53 protein overexpression had been shown to be inversely correlated with MASPIN expression in ovarian cancer [17]. In normal and cancerous breast and prostate cells, wt p53 has been shown to activate MASPIN expression by binding directly to the $p 53$ consensus site in the MASPIN promoter [12]. Oshiro et al. demonstrated that mutant $p 53$ ( $\mathrm{m} \mathrm{p53)}$ and aberrant cytosine methylation cooperate to silence expression of MASPIN [15]. The MASPIN promoter is normally associated with unmethylated cytosines and wt p53 may function to protect target promoters from aberrant methylation through its DNA-binding activity. However, upon mutation the wt p53 DNA-binding, activity is lost and the p53 target regions become permissive to aberrant de novo cytosine methylation which subsequently results in the loss of gene expression [15].

The present study was undertaken to determine the relationship of MASPIN expression and p53 status as well as MASPIN promoter methylation in epithelial ovarian cancer (EOC). The manner in which p53 regulates the expression of MASPIN is poorly understood and may include epigenetic mechanisms such as gene silencing via aberrant cytosine methylation of gene promoters. Our primary hypothesis was that inactivation of the p53 tumor suppressor gene pathway leads to MASPIN repression in ovarian cancers through aberrant promoter hypermethylation.

\section{Methods}

Ovarian cancer cell lines, DNA methyltransferase inhibition, and p53 transfection

\section{Cell culture}

Seven immortalized ovarian cancer cell lines (DOV 13, OVCA 420, OVCA 429, OVCA 432, OVCA 433, SKOV3, OVCAR3) were grown in monolayer culture Type Culture Collection (Manassas, VA) and maintained as recommended by the supplier. The DOV 13, OVCA 420, OVCA 429, and OVCA 433 cell lines contain a wt $p 53$ gene while OVCA 432, OVCAR3, and SKOV3 harbor a m $p 53$ gene [18]. Protein extractions were performed as previously described [18,19] and RNA extractions were performed using the RNeasy Mini Kit following the manufacturer's recommendations (Qiagen, Inc.; Valencia, CA). For cDNA synthesis, $1 \mu \mathrm{g}$ of total RNA was incubated for $60 \mathrm{~min}$ at $42{ }^{\circ} \mathrm{C}$ with oligo (dT) primers and 20 units of AMV reverse transcriptase in $1 \times$ reverse transcriptase buffer supplemented with $5 \mathrm{mM}$ of $\mathrm{MgCl}_{2}$, $1 \mathrm{mM}$ of each dNTP, and 25 units of RNase inhibitor in a final volume of $20 \mu \mathrm{l}$ (Roche Diagnostics Cooperation, Indianapolis, IN). All experiments were performed in triplicate.

\section{DNA methyltransferase inhibition}

Ovarian cancer cell lines were grown in six-well plates and treated for 72 h with $5 \mu \mathrm{M}$ 5-azacytidine (5-azaC; Sigma Aldrich; St. Louis, MO), a potent inhibitor of DNA methyltransferase (DNMT) activity [20], dissolved in dimethylsulfoxide. Controls were treated with dimethylsulfoxide containing media. Cell pellets were divided to extract both protein and RNA to determine MASPIN protein and mRNA expression, respectively.

\section{Transfection experiments}

SKOV3 cells were cultured in $60 \mathrm{~mm}$ plates in RPMI 1640 medium (GIBCO®; Carlsbad, CA) supplemented with $10 \%$ fetal calf serum (FCS) until semi-confluent. Transient co-transfections of SKOV3 cells were performed using Lipofectamine (GIBCO $\left.{ }^{\circledR}\right)$ and OptiMEM. Plasmids used for transfection included: $2 \mu \mathrm{g}$ of pcDNA3 containing wt $p 53$ sequence and corresponding empty vector (provided by Dr. Jeffrey Marks), and $2 \mu \mathrm{g}$ of pEGFP encoding enhanced green fluorescent protein and corresponding empty vector (provided by Dr. Jeffrey Marks). After a $4 \mathrm{~h}$ incubation at $37 \mathrm{C}$, the transfection media was replaced with RPMI 1640 and the cells were incubated at $37 \mathrm{C}$ overnight. Fluorescent microscopy was used to determine transfection efficiency. A subset of the transfected cells was harvested while the remainder underwent treatment for an additional $72 \mathrm{~h}$ with 5-azaC prior to harvest for protein and RNA as described above.

\section{Primary ovarian cancer specimens}

Frozen tumor specimens were obtained during primary cytoreductive surgery and prior to the initiation of chemotherapy from 110 women with advanced EOC, who participated on GOG specimen banking protocol and randomized phase III first-line treatment protocols [21,22]. One patient enrolled on GOG Protocol 114 was inappropriately classified as having optimally-debulked disease; this case was classified as suboptimally-debulked for this translational research study. Tissues were maintained at $-70{ }^{\circ} \mathrm{C}$ and histologic evaluation of each sample was performed to confirm that at least $50 \%$ of the cellular component was malignant. Data regarding immunohistochemical expression of p53 protein and/or sequencing of exons 2-11 or exons 5-8 of the $p 53$ gene and MASPIN protein expression in the primary epithelial ovarian cancers were previously published $[7,23]$.

\section{Western blot analysis and real-time quantitative PCR}

Western blot analysis was performed as previously described [7]. Real-time quantitative PCR (RQ-PCR) was used to analyze mRNA expression in the immortalized ovarian cancer cell lines. Quantification of MASPIN mRNA expression was obtained by RQ-PCR using fluorescent TaqMan methodology (ABI Prism 7700 Sequence Detector; Perkin Elmer Applied BioSystems; Foster City, CA). PCR was performed using $2 \mu \mathrm{l}$ cDNA, $12 \mu \mathrm{l}$ Universal PCR Master Mix (Perkin Elmer Biosystems; Branchburg, NJ, USA), 15 pmol forward and reverse primers specific for each marker and $200 \mathrm{nM}$ specific detection probe in a final volume of $25 \mu \mathrm{l}$. Primers and probes for MASPIN and GAPDH were obtained from Applied Biosystems (Foster City, CA). All PCR reactions were performed on an ABI Prism 7700 Sequence Detector System (Perkin Elmer Applied Biosystems) with a Gene-amp PCR System 9600. The thermal cycling conditions were: $50{ }^{\circ} \mathrm{C}$ for $2 \mathrm{~min}$ and $95^{\circ} \mathrm{C}$ for $10 \mathrm{~min}$ followed by 40 cycles of $95^{\circ} \mathrm{C}$ for $15 \mathrm{~s}$ and $60{ }^{\circ} \mathrm{C}$ for $1 \mathrm{~min}$. The comparative cycle threshold method was used to calculate the relative expression of MASPIN mRNA normalized to GAPDH run in parallel [24].

\section{Methylation analyses}

Bisulfite sequencing to determine the level of MASPIN promoter methylation was performed as previously described [25,26]. The following primers for the initial reaction 5'-AAAGAATGGAGATTAGAGTATTTTTTGTG-3' and 5'-CCTAAAATCACAATTATCCTAAAAAATA3'; and second reaction 5'GAAATTTGTAGTGTTATTATTATTATA-3' and 5'-AAAAACACAAAAACCTAAATATAAAAA-3' were utilized. The product, a 368-bp amplicon, was resolved on $1 \%$ agarose gels, purified using Sigma GenElute gel purification columns (Sigma-Aldrich, St. Louis, MO) and sequenced using the ThermoSequenase Radiolabeled 
Terminator Cycle Sequencing Kit (USB Corporation; Cleveland, $\mathrm{OH}$ ) with primer 5'-TTTTTAATTGTGGATAAGTTGTTAAGAG-3'. Sequencing products were resolved on acrylamide sequencing gels, dried, and exposed to autoradiographic film or to a phosphor screen prior to phosphor imaging using the Molecular Dynamics Storm Phosphor Imaging System (GE Healthcare; Pittsburgh, PA) and ImageQuant software (GE Healthcare). The degree of methylation visualized by autoradiography was scored subjectively from 0 to 4: 0 (unmethylated, $0 \%$ ); 1 (weakly methylated, $>1 \%$ but $<50 \%$ ); 2 (equally methylated, 50\%); 3 (heavily methylated, $>50 \%$ but $<100 \%$ ); and 4 (fully methylated, 100\%). The percent methylation at individual $\mathrm{CpG}$ cytosines measured by phosphor imaging was determined using the formula: percent $\mathrm{mC}=$ volume $\mathrm{C} /($ volume $\mathrm{C}+$ volume $\mathrm{T}) \times 100$.

\section{Statistical analysis}

The biomarker and clinical data for this study were analyzed using SPSS version 10.1 (SPSS Inc., Chicago, IL) or SAS version 8.2 (SAS Institute Inc., Cary NC). Fisher's exact test was used to test the hypothesis of independence between categorical variables in $2 \times 2$ or $\mathrm{r} \times \mathrm{c}$ tables, respectively [27]. The non-parametric Kendall's tau-b correlation coefficient test was used to assess the correlation between MASPIN protein and mRNA [27]. The non-parametric Wilcoxon signedrank test was used to assess the relationship between biomarkers [28]. All tests were two-sided. Mantel-Haenszel analysis was used to evaluate the relationship between MASPIN protein detection to MASPIN promoter methylation and p53 mutation status [27].

\section{Results}

MASPIN expression and promoter methylation status in ovarian cancer cell lines

Lower levels of MASPIN protein and mRNA expression were observed in the $\mathrm{m} p 53$ ovarian cancer cell lines relative to those with wt $p 53$ genes (Fig. 1). The 3 ovarian cancer cell lines with wt p53 expressed 7- to 860-fold higher levels of MASPIN protein compared to cell lines with mutant $p 53$. A non-parametric Wilcoxon signed-rank test provided suggestive evidence of a trend between $p 53$ mutation and low levels of MASPIN protein or mRNA $(\mathrm{p}=0.057)$. The ovarian cancer cell lines were divided into 2 groups based on the level of MASPIN protein expression. Of the 3 MASPIN-low $\mathrm{m}$ p53 cell lines 2 had greater than $5 \%$ MASPIN methylation whereas only 1 of 4 MASPINhigh wt $p 53$ cell lines had greater than 5\% MASPIN methylation (Fig. 1). RQ-PCR also demonstrated higher levels of MASPIN mRNA in the wt p53 cell lines compared to the mutant p53 cell lines (Fig. 1). MASPIN protein and mRNA were highly correlated (Kendall's tau-b correlation coefficient $=0.8, \mathrm{p}<0.001)$.

Effects of demethylating agent 5-azacytidine and p53 transfection on MASPIN promoter methylation and transcription in ovarian cancer cell lines

After treatment with 5-azaC, two MASPIN-low cell lines, OVCAR3 and SKOV3, demonstrated a 2.7- and 2.3-fold induction, respectively, of MASPIN transcription as compared to mock-treated controls (Fig. 2A). Following 5-azaC treatment OVCAR3 was completely demethylated while SKOV3 had only a minimal decrease in promoter methylation (Fig. 2B). In contrast, the MASPIN-high cell lines demonstrated no appreciable change in MASPIN transcription or MASPIN methylation after treatment with 5-azaC (Figs. 2A and B).

Despite the presence of aberrant MASPIN promoter methylation in SKOV3 cells, wt p53-transfection alone resulted in a 3.3-fold increase in MASPIN mRNA (Fig. 2C). However, there was no reduction in MASPIN promoter methylation following wt p53 introduction (Fig. 2D). But when wt $p 53$ transfection was combined with 5-azaC therapy, MASPIN
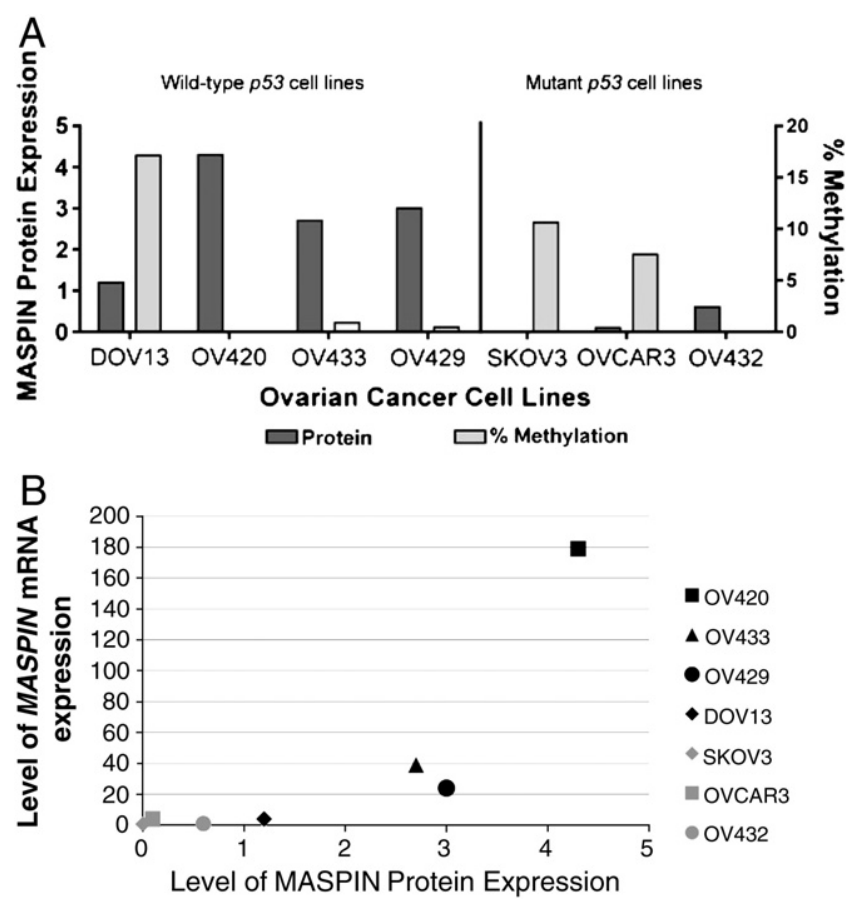

Fig. 1. MASPIN promoter methylation, mRNA and protein expression in the ovarian cancer cell lines. (A) The four ovarian cancer cell lines with wt p53 are denoted on the left and demonstrate higher levels of MASPIN protein expression and 3 exhibit low levels of MASPIN promoter methylation. In contrast the three ovarian cancer cell lines harboring a p53 mutation demonstrated low MASPIN protein expression and were more likely to display greater MASPIN promoter methylation. The percent methylation at individual $\mathrm{CPG}$ cytosines of the MASPIN promoter was scored objectively by phosphorimaging for DOV13, OV429, OV433, SKOV3, and OVCAR3. MASPIN promoter methylation was determined subjectively in the OV420 and OV432 cell lines. (B) MASPIN mRNA and protein expression were highly correlated in the ovarian cancer cell lines. High MASPIN mRNA and protein expression were seen in the wt $p 53$ cell lines (black shapes) while the mutant $p 53$ cell lines (gray shapes) demonstrated low MASPIN expression.

promoter methylation decreased 36\% (Fig. 2D) and this was accompanied by a 4.5 -fold increase in MASPIN transcription (Fig. 2C).

MASPIN expression and correlation with p53 status and MASPIN methylation status in ovarian cancer specimens

MASPIN expression was not associated with the level of p53 overexpression categorized as no overexpression, limited overexpression ( $<30 \%$ tumor cells exhibiting p53 immunostaining) or extensive overexpression ( $>30 \%$ tumor cells displaying p53 immunostaining) $(p=0.12)$ or extensive $p 53$ overexpression compared with no or limited overexpression $(\mathrm{p}=0.09$ ) (Table 1$)$. However, evidence of an association was observed between MASPIN expression and the type of p53 mutation categorized as no mutation, missense mutation, or a truncation mutation in exons $2-11(p=0.032)$, but not in exons 5 $8(p=0.123)$ of the $p 53$ gene. Further exploratory analysis revealed that detectable MASPIN was preferentially associated with a missense mutation compared with no mutation or a truncation mutation in exons $2-11$ of $p 53(p=0.012)$. Eighty-six percent of cancers harboring a missense mutation in exons $2-11$ exhibited detectable MASPIN whereas only $53 \%$ and $57 \%$ of those with wt p53 or a truncation mutation in exons 2-11 demonstrated detectable MASPIN, respectively.

The MASPIN promoter was weakly methylated in $81.8 \%(90 / 110)$, heavily methylated in $14.5 \%$ (16/110), and fully methylated in $3.6 \%$ (4/110) of the specimens (Fig. 3). Data regarding both MASPIN protein expression and promoter methylation were available for 66 patients and data regarding both MASPIN protein expression and $p 53$ mutation status was available for 104 patients. Fisher's exact testing was used to 
A

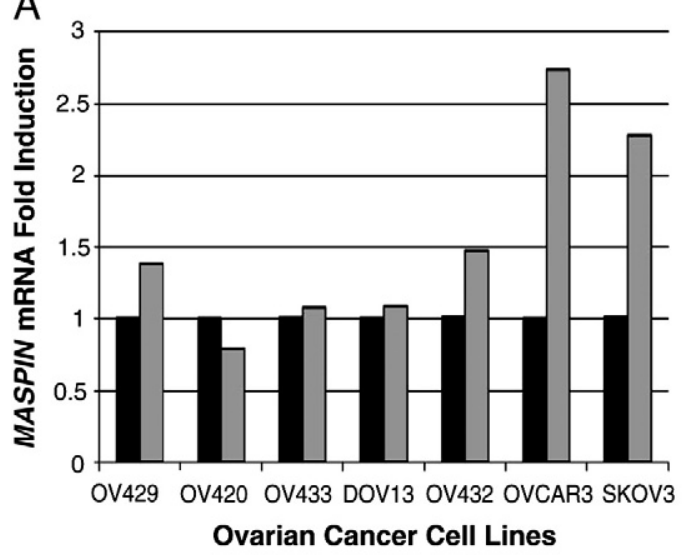

C

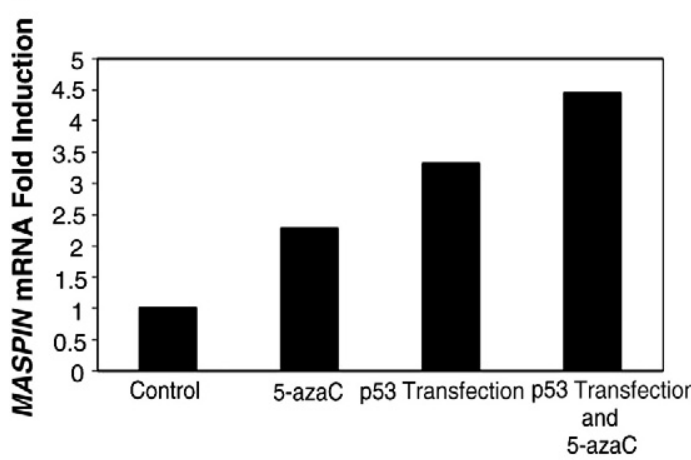

Treatment
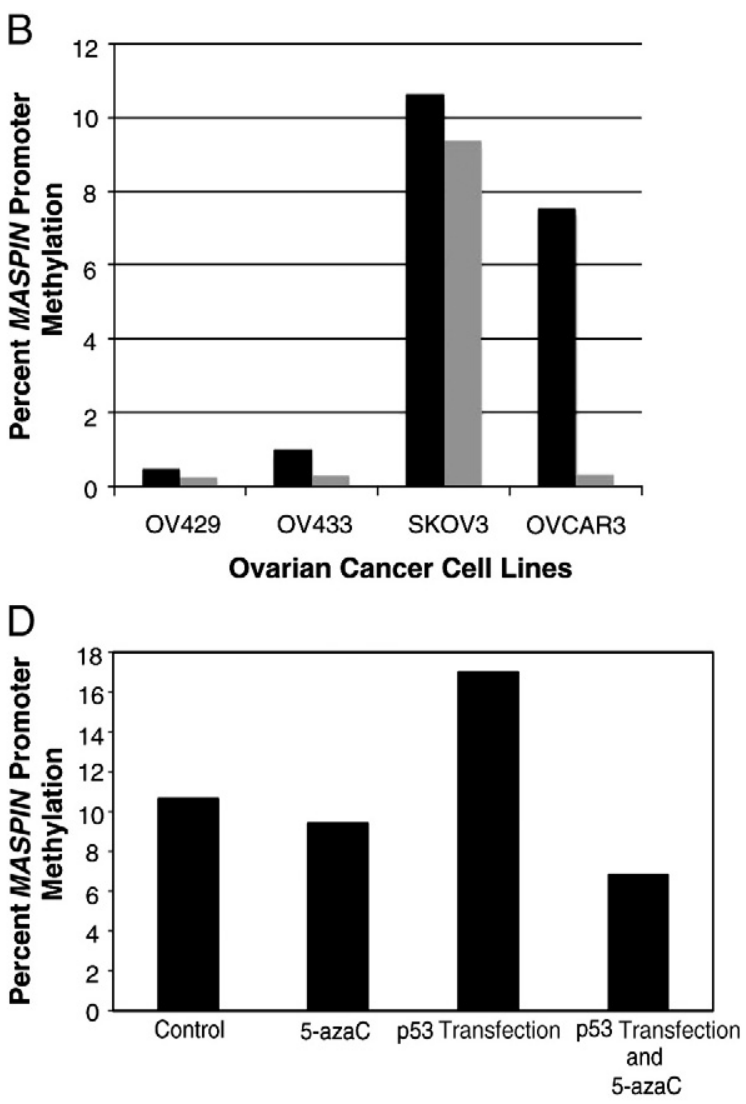

Treatment

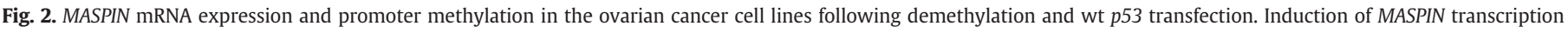

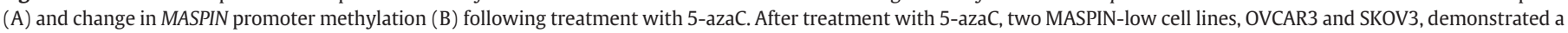

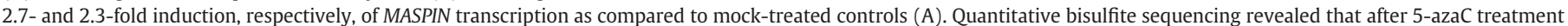

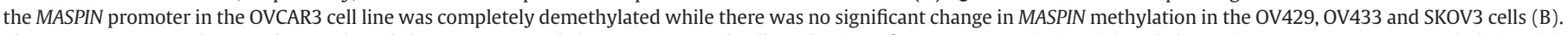

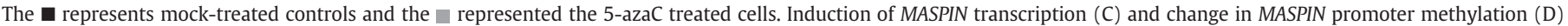
following treatment with either 5-azaC, transfection of a plasmid encoding wt p53, or treatment with 5-azaC plus introduction of wt $p 53$ in SKOV3 cells.

Table 1

Relationship between MASPIN espression and p53 status.

\begin{tabular}{|c|c|c|c|c|c|}
\hline \multirow[b]{3}{*}{ p53 alterations } & \multicolumn{4}{|c|}{ Categorized MASPIN expression } & \multirow[b]{3}{*}{ p-value } \\
\hline & \multicolumn{2}{|c|}{$\begin{array}{l}\text { Non- } \\
\text { detectable }\end{array}$} & \multicolumn{2}{|c|}{ Detectable } & \\
\hline & No. & $(\%)$ & No. & $(\%)$ & \\
\hline \multicolumn{6}{|l|}{ Overexpression of p53 $(n=62)^{a}$} \\
\hline No & 8 & $(36.4)$ & 14 & $(63.6)$ & $0.12^{\mathrm{CA}}$ \\
\hline Limited $^{\mathrm{b}}$ & 4 & $(40.0)$ & 6 & $(60.0)$ & \\
\hline Extensive $^{\mathrm{b}}$ & 5 & $(16.7)$ & 25 & $(83.3)$ & \\
\hline No or limited ${ }^{\mathrm{b}}$ & 12 & $(37.5)$ & 20 & $(62.5)$ & $0.09^{\mathrm{FE}}$ \\
\hline Extensive $^{\mathrm{b}}$ & 5 & $(16.7)$ & 25 & $(83.3)$ & \\
\hline \multicolumn{6}{|c|}{ Mutation in exons $2-11$ of $p 53(\mathrm{n}=59)^{\mathrm{a}}$} \\
\hline No mutation & 8 & $(47.1)$ & 9 & $(52.9)$ & $0.032^{\mathrm{FE}}$ \\
\hline Missense & 4 & $(14.3)$ & 24 & $(85.7)$ & \\
\hline Truncation & 6 & $(42.9)$ & 8 & $(57.1)$ & \\
\hline No mutation or truncation & 14 & $(45.2)$ & 17 & $(54.8)$ & $0.012^{\mathrm{FE}}$ \\
\hline Missense & 4 & $(14.3)$ & 24 & $(85.7)$ & \\
\hline \multicolumn{6}{|c|}{ Mutation in exons $5-8$ of $p 53(n=68)^{a}$} \\
\hline No mutation & 11 & $(34.4)$ & 21 & $(65.6)$ & $0.123^{\mathrm{FE}}$ \\
\hline Missense & 4 & $(14.8)$ & 23 & $(85.2)$ & \\
\hline Truncation & 4 & $(14.8)$ & 5 & $(55.6)$ & \\
\hline No mutation or truncation & 15 & $(36.6)$ & 26 & $(63.4)$ & $0.059^{\mathrm{FE}}$ \\
\hline Missense & 4 & (14.8) & 23 & $(85.2)$ & \\
\hline
\end{tabular}

CA: Cochran-Armitage exact trend test; FE: Fisher's exact test.

a Immunoblot data for MASPIN was available for 62 cases with previously published immunohistochemical staining results for $\mathrm{p} 53$, for 59 cases with previously published mutation data within exons $2-11$ of $p 53$, and for 68 cases with previously published mutation data within exons $5-8$ of $p 53$.

b Limited overexpression: $<30 \%$ p53 positive tumor cells; extensive overexpression: $>30 \%$ p53 positive tumor cells. examine the association between relative MASPIN expression categorized as non-detectable or detectable, and MASPIN promoter methylation and alterations in p53 (Table 2). There was no relationship between promoter methylation and p53 status or MASPIN protein expression in these primary ovarian cancers ( $p$-value $=0.55$ and 0.265 ). However, tumors harboring alterations of $p 53$ were more likely to have detectable MASPIN protein expression (Table 3). MASPIN protein was 6 times more likely to be detected in cancer specimens that harbor a $p 53$ mutation relative to cancer specimens with an intact wt $p 53$ gene.

\section{Discussion}

Investigators have published evidence to suggest that MASPIN is regulated by $p 53$ in a number of solid tumors including breast, prostate, colon, and ovarian cancers $[12,17,29,30]$. Our data from immortalized ovarian cell lines, although limited, are consistent with the paradigm that MASPIN is regulated via both p53-dependent and independent pathways. The ovarian cancer cell lines harboring mutant p53 genes expressed low MASPIN protein and mRNA levels and were more likely to have increased MASPIN promoter methylation. In contrast, ovarian cancer cell lines with wt $p 53$ expressed high MASPIN protein levels and were more likely to display decreased or absent MASPIN promoter methylation.

Our finding that MASPIN promoter demethylation occurred upon treatment with 5-azaC in the OVCAR3 cell line with a p53 missense mutation but not in the SKOV3 cell line, which harbors a p53 truncating mutation indicate that the relationship between $p 53$ and 
0

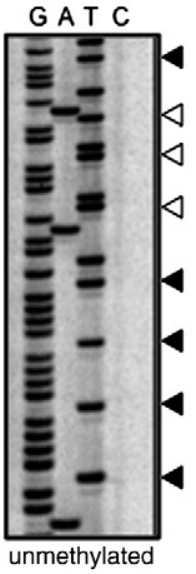

1

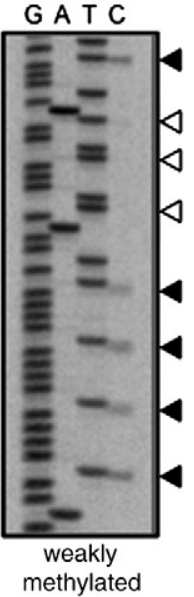

2

G A T C

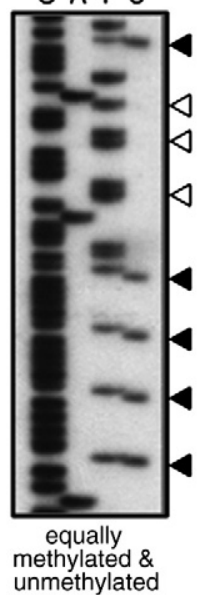

3

G A T C

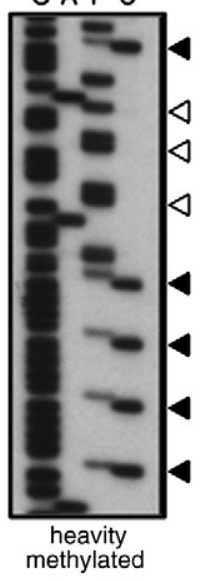

4

G A T C

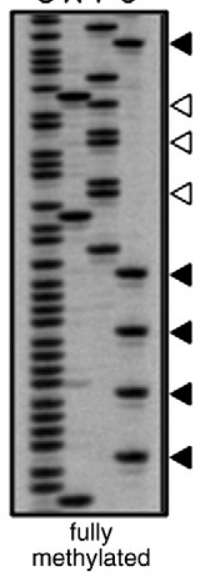

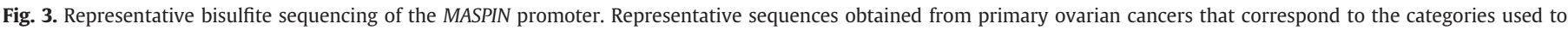

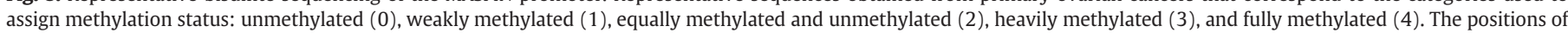

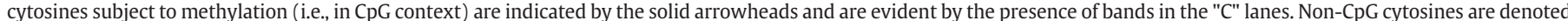

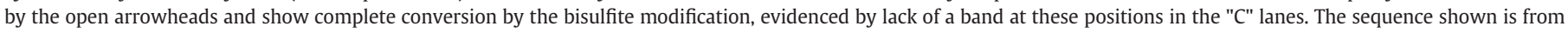
-44 bp upstream to +10 bp downstream of the annotated transcription start site of MASPIN (reference sequence NM_002639.4).

aberrant cytosine methylation may be $p 53$ mutation-type dependent. Furthermore, MASPIN mRNA transcription was independently reactivated through inhibition of DNMT activity or through introduction of wt $p 53$ into SKOV3 cells. However, forced wt $p 53$ introduction was not capable of reversing MASPIN promoter methylation in SKOV3 cells, suggesting that wt $p 53$ may reactivate MASPIN gene transcription via alternative pathways other than promoter demethylation and that wt p53 alone cannot modify the methylation status of the promoter. It is presently unknown what specific percent change in methylation induces a specific quantitative change in transcription. Our data show that a small change in methylation, at least in the SKOV3 cell line, is associated with induction of MASPIN transcription. Whether or not the induction is due entirely to this decrease in methylation and/or to methylation changes at other regions of the MASPIN promoter, or to upstream effects, is not known at this time. Variable responses to DNMT inhibitors (DOV13 and OV432) were seen that may reflect the status of histone modifications and resulting chromatin structure and/ or less efficient uptake or incorporation of the cytosine analog into the DNA. Induction of expression in the absence of promoter methylation in the case of OV432 is very likely attributable to indirect effects of the 5 -azaC treatment whereby, for example, an upstream transcription factor is reactivated by the treatment, and in turn is able to activate expression of its downstream targets.

Table 2

Relationship between MASPIN promoter methylation and MASPIN protein expression and $p 53$ mutations.

\begin{tabular}{llll}
\hline & \multicolumn{2}{l}{ Level of MASPIN promoter methylation } & \multirow{2}{*}{ p-value } \\
\cline { 2 - 3 } & $\begin{array}{l}\text { Un/weakly/equally } \\
\text { methylated }(0,1,2)\end{array}$ & $\begin{array}{l}\text { Heavily/fully } \\
\text { methylated }(3,4)\end{array}$ & \\
\hline \multicolumn{2}{l}{ MASPIN protein expression ${ }^{\text {a }}$} & & \\
$\quad \begin{array}{l}\text { Non-detectable } \\
\text { Detectable }\end{array}$ & $15(23 \%)$ & $3(4 \%)$ & 0.55 \\
p53 mutation status & $41(62 \%)$ & $7(11 \%)$ & \\
$\quad$ Mutated & $59(57 \%)$ & $12(11 \%)$ & 0.265 \\
$\quad$ Wild-type & $25(24 \%)$ & $8(8 \%)$ & \\
\hline
\end{tabular}

a Data regarding both MASPIN protein expression and promoter methylation was available on 66 patients.

${ }^{\mathrm{b}}$ Data regarding both MASPIN protein expression and $p 53$ mutation status was available on 104 patients.
Our findings regarding the SKOV3 transfection experiments are consistent with those reported by Oshiro and Murakami [15,31] whereby reintroduction of $p 53$ partially reactivated MASPIN genes expression. However, wt $p 53$ did not affect the methylation status of the promoter, signifying that wt $p 53$ itself can only partially overcome the repressive barrier of DNA methylation. $p 53$ restoration combined with demethylation via 5-azaC synergistically restored MASPIN expression in the breast cancer cell lines to levels of expression approaching the basal levels seen in an immortalized, non-tumorigenic breast epithelial cancer cell line [15]. Similarly, our data revealed an additive effect of demethylation and forced wt $p 53$ reintroduction on MASPIN expression in the SKOV3 ovarian cancer cells. Oshiro also found that wt $p 53$ binding to the MASPIN promoter DNA-binding site stimulated histone acetylation and enhanced accessibility of the promoter site. Changes in histone acetylation can affect the chromatin structure, increasing accessibility for transcription and thus play an important role in gene expression [32]. This mechanism of action may explain how p53 reintroduction induces MASPIN expression despite the presence of DNA methylation. In summary, these results suggest that alterations of $p 53$ and aberrant DNA methylation may control MASPIN gene transcription via independent temporal events but interconnected synergistic events that affect MASPIN expression.

Our findings do differ from that of Rose and colleagues who evaluated MASPIN expression and promoter methylation in normal

Table 3

Relationship between MASPIN promoter methylation, p53 mutation status or p53 immunohistochemical (IHC) expression and non-detectable MASPIN protein in primary ovarian cancers.

\begin{tabular}{lrrlll}
\hline & \multicolumn{5}{c}{ Non-detectable MASPIN protein } \\
\cline { 2 - 6 } & Yes & No & OR & $95 \%$ CI & p-value \\
\hline MASPIN methylation & & & & & \\
Un/weakly/equally (0, 1 or 2) & 15 & 41 & Referent & & \\
Heavily/fully (3 or 4) & 3 & 7 & 0.854 & $0.195-3.736$ & 0.834 \\
p53 missense mutation in exons $2-11$ & & & & \\
$\quad$ No & 14 & 17 & Referent & & \\
Yes & 4 & 24 & 4.941 & $1.383-17.649$ & 0.014 \\
p53 IHC expression & & & & & \\
$\quad$ Negative & 10 & 16 & Referent & & \\
$\quad$ Positive & 9 & 32 & 2.222 & $0.753-6.558$ & 0.148 \\
\hline
\end{tabular}

Mantel-Haenszel common odds ratio (OR) estimate and asymptotic 95\% confidence interval $(\mathrm{CI})$. 
human ovarian surface epithelium (HOSE) and three ovarian cancer cell lines; two harboring $\mathrm{m} p 53$ genes (A222 a silent mutation and OVCAR3 a missense mutation) and a SKOV3 cell line with wt p53 gene [33]. They reported that the HOSE and A222 cell lines were MASPIN-negative and had highly methylated MASPIN promoters (60\% and 88\%, respectively). In contrast, the MASPIN-positive cancer cell lines, OVCAR3 and SKOV3, had $<0.5 \%$ and $40 \%$ MASPIN promoter methylation, respectively, which the authors considered undermethylated. The level of MASPIN promoter methylation and protein expression in the SKOV3 cell lines varied between our studies and may be attributable to the disparity in $p 53$ status. Rose and colleagues used SKOV3 cells with wt p53 that may account for the detection of MASPIN protein if p53 was able to transcriptionally induce MASPIN despite the presence of promoter DNA methylation. In contrast, our MASPIN-negative SKOV3 cells also exhibited MASPIN promoter methylation but this was in the context of a m p53 and are consistent with the findings of Zhang et al. [17]. The diverse findings between the studies may be related to differences in methodology, cell lines, historical differences in culture conditions, and the $p 53$ mutational status of the SKOV3 cell line.

Based on our in vitro data, we predicted that ovarian cancers with a p53 mutation would have non-detectable MASPIN protein expression whereas those with no mutation would exhibit detectable MASPIN. In contrast, $86 \%$ of the ovarian cancers with a missense mutation within exons 2-11 of $p 53$ displayed detectable MASPIN, while $53 \%$ of cancers with no mutation exhibited detectable MASPIN. Further analysis revealed that MASPIN protein was 6 times more likely to be detected in cancer specimens that harbor a p53 mutation relative to cancer specimens with an intact wt p53 gene. In addition there was no association between MASPIN promoter methylation and $p 53$ status or MASPIN protein expression. A lack of association between promoter methylation and protein expression can be seen if mRNA expression and protein levels are dissociated. However, our in vitro data do not support dissociated MASPIN transcription and translation. Disparity in the results obtained in cell lines compared with clinical tumor specimens has been previously reported in studies of other molecular pathways in immortalized ovarian cancer cell lines compared with primary ovarian cancers $[18,34,35]$. Immortalized ovarian cancer cell lines may not be representative of primary cancers with respect to regulation of growth and angiogenesis. Regardless our results in the tumor specimens do not support that methylation silencing of the MASPIN promoter is controlled by p53 or regulated MASPIN expression. Our results may have been limited by the small number of specimens evaluated.

Others have reported that MASPIN may be regulated via p53independent mechanisms such as E-twenty six (ETS) and activator protein-1 (AP-1) transcription site activation, hormonal responsive element site repression $[9,10]$, and by the $p 63$ pathway [16]. Our data indicate that the regulation of MASPIN is a complex multifactorial process, that may be controlled by both epigenetic and genetic mechanisms.

Conflict of interest

The co-authors have no conflicts of interest to declare.

\section{References}

[1] Zou Z, Anisowicz A, Hendrix MJ, Thor A, Neveu M, Sheng S, et al. Maspin, a serpin with tumor-suppressing activity in human mammary epithelial-cells. Science 1994;263:526-9.

[2] Zhang M, Volpert O, Shi YH, Bouck N. Maspin is an angiogenesis inhibitor. Nat Med 2000;6:196-9.

[3] Hopkins PC, Whisstock J. Function of maspin. Science 1994;265:1893-4.

[4] Sheng S, Carey J, Seftor EA, Dias L, Hendrix MJ, Sager R. Maspin acts at the cell membrane to inhibit invasion and motility of mammary and prostatic cancer cells. Proc Natl Acad Sci USA 1996;93:11669-74.

[5] Solomon LA, Munkarah AR, Schimp VL, Arabi MH, Morris RT, Nassar H, et al. Maspin expression and localization impact on angiogenesis and prognosis in ovarian cancer. Gynecol Oncol 2006;101:385-9.

[6] Sood AK, Fletcher MS, Gruman LM, Coffin JE, Jabbari S, Khalkhali-Ellis Z, et al. The paradoxical expression of maspin in ovarian carcinoma. Clin Cancer Res 2002;8: 2924-32.
[7] Secord AA, Lee PS, Darcy KM, Havrilesky LJ, Grace LA, Marks JR, et al. Maspin expression in epithelial ovarian cancer and associations with poor prognosis: a Gynecologic Oncology Group study. Gynecol Oncol 2006;101:390-7.

[8] Ito R, Nakayama H, Yoshida K, Oda N, Yasui W. Loss of maspin expression is associated with development and progression of gastric carcinoma with p53 abnormality. Oncol Rep 2004;12:985-90.

[9] Zhang M, Magit D, Sager R. Expression of maspin in prostate cells is regulated by a positive Ets element and a negative hormonal responsive element site recognized by androgen receptor. Proc Natl Acad Sci USA 1997;94:5673-8.

[10] Zhang M, Maass N, Magit D, Sager R. Transactivation through Ets and Ap1 transcription sites determines the expression of the tumor-suppressing gene maspin. Cell Growth Differ 1997;8:179-86.

[11] Domann FE, Rice JC, Hendrix MJ, Futscher BW. Epigenetic silencing of maspin gene expression in human breast cancers. Int J Cancer 2000;85:805-10.

[12] Zou ZQ Gao CL, Nagaich AK, Connell T, Saito S, Moul JW, et al. p53 regulates the expression of the tumor suppressor gene maspin. J Biol Chem 2000;275:6051-4.

[13] Costello JF, Vertino PM. Methylation matters: a new spin on maspin. Nat Genet 2002;31:123-4.

[14] Fitzgerald M, Oshiro M, Holtan N, Krager K, Cullen JJ, Futscher BW, et al. Human pancreatic carcinoma cells activate maspin expression through loss of epigenetic control. Neoplasia 2003;5:427-36.

[15] Oshiro MM, Watts GS, Wozniak RJ, Junk DJ, Munoz-Rodriguez JL, Domann FE, et al. Mutant p53 and aberrant cytosine methylation cooperate to silence gene expression. Oncogene 2003;22:3624-34.

[16] Kim S, Han JH, Kim J, Park C. Maspin expression is transactivated by p63 and is critical for the modulation of lung cancer progression. Cancer Res 2004;64: 6900-5.

[17] Zhang W, Zhang M. Tissue microarray analysis of maspin expression and its reverse correlation with mutant p53 in various tumors. Int J Oncol 2002;20: 1145-50.

[18] Havrilesky LJ, Alvarez AA, Whitaker RS, Marks JR, Berchuck A. Loss of expression of the p16 tumor suppressor gene is more frequent in advanced ovarian cancers lacking p53 mutations. Gynecol Oncol 2001;83:491-500.

[19] Bernardini MQ Baba T, Lee PS, Barnett JC, Sfakianos GP, Secord AA, et al. Expression signatures of TP53 mutations in serous ovarian cancers. BMC Cancer 2010;10: 237-46.

[20] Primeau M, Gagnon J, Momparler RL. Synergistic antineoplastic action of DNA methylation inhibitor 5-AZA-2'-deoxycytidine and histone deacetylase inhibitor depsipeptide on human breast carcinoma cells. Int J Cancer 2003;103:177-84.

[21] Markman M, Bundy BN, Alberts DS, Fowler JM, Clark-Pearson DL, Carson LF, et al. Phase III trial of standard-dose intravenous cisplatin plus paclitaxel versus moderately high-dose carboplatin followed by intravenous paclitaxel and intraperitoneal cisplatin in small-volume stage III ovarian carcinoma: an intergroup study of the Gynecologic Oncology Group, Southwestern Oncology Group, and Eastern Cooperative Oncology Group. J Clin Oncol 2001;19:1001-7.

[22] Muggia FM, Braly PS, Brady MF, Sutton G, Niemann TH, Lentz SL, et al. Phase III randomized study of cisplatin versus paclitaxel versus cisplatin and paclitaxel in patients with suboptimal stage III or IV ovarian cancer: a Gynecologic Oncology Group study. J Clin Oncol 2000;18:106-15.

[23] Havrilesky L, Darcy KM, Hamdan H, Priore RL, Leon J, Bell J, et al. Prognostic significance of p53 mutation and p53 overexpression in advanced epithelial ovarian cancer: a Gynecologic Oncology Group Study. J Clin Oncol 2003;21: 3814-25.

[24] Schmittgen TD, Livak KJ. Analyzing real-time PCR data by the comparative C(T) method. Nat Protoc 2008;3:1101-8.

[25] Huang Z, Wen Y, Shandilya R, Marks JR, Berchuck A, Murphy SK. High throughput detection of M6P/IGF2R intronic hypermethylation and LOH in ovarian cancer. Nucleic Acids Res 2006;34:555-63.

[26] Futscher BW, Ohiro MM, Wozniak RJ, Holtan N, Hanigan CL, Duan H, et al. Role for DNA methylation in the control of cell type-specific maspin expression. Nat Genet 2002;31:175-9.

[27] Agresti A. Categorical data analysis. Wiley Series in Probability and Statistics, New York, NY; 2002. p. 34. 60-4; 230-4.

[28] Lehmann EL. Nonparametrics: statistical methods based on ranks. New York, NY: Springer Science; 2006. p. 123-32.

[29] Machtens S, Serth J, Bokemeyer C, Bathke W, Minssen A, Kollmannsberger C, et al. Expression of the $\mathrm{p} 53$ and maspin protein in primary prostate cancer: correlation with clinical features. Int J Cancer 2001;95:337-42.

[30] Song SY, Lee SK, Kim DH, Son HJ, Kim HJ, Lim YJ, et al. Expression of maspin in colon cancers - its relationship with p53 expression and microvessel density. Dig Dis Sci 2002;47:1831-5.

[31] Murakami J, Asaumi J, Maki Y, Tsujigiwa H, Kuroda M, Nagai N, et al. Effects of demethylating agent 5-aza-2'-deoxycytidine and histone deacetylase inhibitor FR901228 on maspin gene expression in oral cancer cell lines. Oral Oncol 2004;40: 597-603.

[32] Hebbes TR, Thorne AW, Crane-Robinson CA. A direct link between core histone acetylation and transcriptionally active chromatin. EMBO J 1988;7:1395-402.

[33] Rose SL, Fitzgerald MP, White NO, Hitchler MJ, Futscher BW, De Geest K, et al. Epigenetic regulation of maspin expression in human ovarian carcinoma cells. Gynecol Oncol 2006;102:319-24.

[34] Berchuck A, Rodriguez GC, Olt GJ, Boente MP, Whitaker RS, Arrick B, et al. Regulation of growth of normal ovarian epithelial cells and ovarian cancer cell lines by transforming growth factor-b. Am J Obstet Gynecol 1992;166:676-84.

[35] Hurteau J, Rodriguez GC, Whitaker RS, Shah S, Mills G, Bast RC, et al. Transforming growth factor-beta inhibits proliferation of human ovarian cancer cells obtained from ascites. Cancer 1994;74:93-9. 